\title{
The Pan-STARRS1 Static Sky: A Classical Physics Goldmine for Cosmology, Astrophysics and Atomic Physics
}

\author{
Ogaba Philip Obande ${ }^{1}$ \\ ${ }^{1}$ Department of Chemistry, Ahmadu Bello University, Zaria, Nigeria (Retired) \\ Correspondence: Ogaba Philip Obande, Department of Chemistry, Ahmadu Bello University, Zaria, Nigeria. \\ E-mail: gababands@gmail.com
}

Received: October 6, 2017

Accepted: October 25, 2017

Online Published: November 30, 2017

doi:10.5539/apr.v9n6p75

URL: https://doi.org/10.5539/apr.v9n6p75

\begin{abstract}
Image of the entire sky, the "Static Sky" recently released to the public by scientists at the Pan-STARRS1 telescope in Hawaii is analyzed. It depicts, for the very first time, nature's intrinsic wave-particle duality; the horizontal finegrained darker envelope is nature's waveform, i.e., the imponderable bosonic cosmic vacuum field, while the brighter coarser-grained vertical envelope belongs to aggregate radiation of visible and invisible particulate matter (fermionic) fields. Earlier investigations were cited to inform that: i) corresponding bosonic field envelope encloses every particulate matter exactly as shown in the Static Sky image; ii) reprocessing the image into a brighter shade reveals the vertical envelope to comprise three bands with diminishing brightness from the center attributed to three ref. frames or universes with common chemical periodicity in line with previous reports on the subject; iii) the visible universe is bifurcated, the effect gives rise to its proximate linear atomic mass growth rate as against logarithmic growth rates in the invisible universes; iv) the two main observational evidences supporting the Big Bang model are faulted on fundamental theoretical grounds which show that: a) cosmological redshift phenomena arise from axial not radial motion, the effect is created by the vacuum field parametric self-interaction $\rho_{\mathrm{w}} / \sigma_{\mathrm{w}}=8.5114 \times 10^{-19}(\mathrm{~m} \mathrm{rad} / \mathrm{s})^{-2}$ whose dimensional analysis yields the superluminal angular velocity $\mathrm{V}_{\mathrm{W}}=4.709 \times 10^{8} \mathrm{~m} / \mathrm{s} ; \mathrm{b}$ ) the cosmic microwave background CMB radiation is classical zero-point radiation whose temperature easily evaluates from summation of bosonic transverse fields' energies $E_{w} / J=h \vartheta_{w}$ of the chemical elements, $\mathrm{T}_{\text {vac. }}=\sum_{\mathrm{e}}^{\mathrm{Am}} \mathrm{E}_{\mathrm{W}} / \mathrm{k}=3.675 \times 10^{-23} \mathrm{~J} / 1.381 \times 10^{-23} \mathrm{~J} / \mathrm{K}=1.662 \mathrm{~K} ; \mathrm{v}$ ) resolution of the vertical envelope into three bands of diminishing brightness indicates that material composition of the two invisible universes, i.e., "dark matter" is accessible with the techiques employed by the Pan-STARRS' scientists.
\end{abstract}

Keywords: big bang model, classical atomic physics, cosmic microwave background, metric space expansion, pan-starrs static sky

\section{Introduction}

Scientists at the Pan-STARRS telescope in Hawaii released to the public a unique image of the visible universe which they call "Static Sky", credited to Danny Farro Science Consortium and Max Planck Institute for Extraterrestrial Physics; New Atlas published the image in 2016. In our opinion the "Static Sky" has the potential to resolve all, pending and yet to be contemplated, questions in physics. Here, we try to explain what we think gives rise to the image and why we have the highest expectation of its potential for unprecedented revolution in classical quantum physics, astrophysics and cosmology.

\section{Method}

We will use methods which we have reported severally to support our explanation of the image and suggest ways to harness its immensely rich potentials; see Obande (2013, 2015a, 2015b, 2015c, 2016a) for detailed descriptions of the methods.

\section{Results}

We illustrate some of our positions with a set of five figures: Figure 1 is, of course, a reproduction of the "Static Sky" as made public by Newatlas.com (2016), Figure 2 is an illustration of mutual orthogonality of frames of reference or universes that work in harmony to create in us a profound experience of reality, Figure 3 illustrates the bifurcation of our visible universe without which our experience of reality would be entirely different and Figs. 4 and 5 illustrate the causality of radioactivity, a common feature of every particulate matter. 


\section{Discussion}

If you try to simulate observational relative atomic mass $m_{r}$ values using the classical mass formula $\mathrm{m}=\mathrm{h} \vartheta / \mathrm{c}^{2}$ you get values that do not seem to relate to reality; they start with $7.3725 \times 10^{-51} \mathrm{~kg} /$ atom for $\vartheta=1.0 \mathrm{~Hz}$ and rise to m $=4.7432 \times 10^{-38} \mathrm{~kg} /$ atom for $\vartheta=6.4425 \times 10^{9} \mathrm{~Hz}$ for americium which marks the end of the natural periodicity; these values contrast with approximate linear growth rate and two orders of magnitude difference between empirical relative atomic mass values of $\mathrm{H}$ and $\mathrm{Am}$. Thus, use of the classical mass formula yields quantum mass values of the atomic waveform, they follow a logarithmic function with about 12 orders of magnitude difference between $\mathrm{H}$ and Am; we attribute these ultra low rest mass values, of course, to "absolute atomic mass" $\mathrm{m}_{\mathrm{abs}}$ or simply $\mathrm{m}_{\mathrm{w}}$. If you now divide $\vartheta$ or $\mathrm{m}$ values of the elements by $\mathrm{H}$ atom's value you get theoretical $\mathrm{m}_{\mathrm{r}}$ values, they correspond to empirical values only up to He from which you observe increasing deviation from empirical values with an increase in atomic number. In order to realize empirical values, you may have to employ a semi-empirical model that produces a linear function from appropriate combinations of two exponential functions. It will yield a final result that makes a compelling case for existence of three ref. frames or universes which work in harmony to produce a common experience of reality; they include: i) an Absolute universe or ref. frame $\mathrm{U}^{*}{ }_{\mathrm{w}}$ that comprises only elemental waveforms; ii) a particulate matter component of the Absolute universe or ref. frame $U_{p}^{*}$; iii) our visible condensed matter universe $\mathrm{U}_{\mathrm{p}}^{\circ}$, and iv) an invisible particulate matter analogue of our visible universe $\mathrm{U}_{\mathrm{p}}^{\prime}$. Notably, all four universes identify with a common periodicity of the chemical elements; in other words, each chemical element exists in four forms comprising one microcosmic waveform and three particulate matter forms; physics identifies the condensed matter forms with "particle generations" Francis (2015).

In order to unambiguously identify the first element of the chemical periodicity, call it E1, you will adopt the convention that reduces absolute atomic mass values to unit $\mathrm{H}$ atom for which we have $\mathrm{m}_{\mathrm{abs}(\mathrm{H})}=1.5078 \times 10^{-47}$ $\mathrm{kg} / \mathrm{u}$ and $\vartheta_{\mathrm{abs}(\mathrm{H})}=2048 \mathrm{~Hz}$. The values give $\mathrm{m}_{\mathrm{r}(\mathrm{E} 1)}=1 \times 10^{-3} / 2048=4.8828125 \times 10^{-7}=7.3725 \times 10^{-51} / 1.5078 \times 10^{-}$ ${ }^{44}=4.8896 \times 10^{-7} \mathrm{~kg} / \mathrm{u}$. From closeness of the results to electron's empirical relative atomic mass, $\mathrm{m}_{\mathrm{r}(\mathrm{e})}=5.4858$ $\mathrm{x} 10^{-7} \mathrm{~kg} / \mathrm{u}$, you cannot but conclude that $\mathrm{E} 1=\mathrm{e}$, the electron. In other words, you find amazingly that there exists a "natural" periodicity of the chemical elements that accords e full-fledged elemental status and identifies it with premier position. The line of investigation leads you to discover 27 unknown elements that seamlessly add to the conventional periodicity - that is a summary of results of our investigations, Obande (2013, 2015a, 2016a); it leads to the unavoidable conclusion that: i) reality comprises a universe made up entirely of waveforms and ii) two invisible particulate matter universes work in harmony to produce our visible matter universe. We now present the "Static Sky".

\subsection{The Static Sky is the Cosmic Large Scale}

Newatlas.com's (2016) publication of the "Static Sky" is reproduced in Figure 1, it comprises two distinct spacetime envelopes, a vertical granular brighter envelope is encased in a finer darker horizontal envelope. We submit that the image presents, for the very first time, a visual evidence of nature's intrinsic wave-particle duality; we review the details.

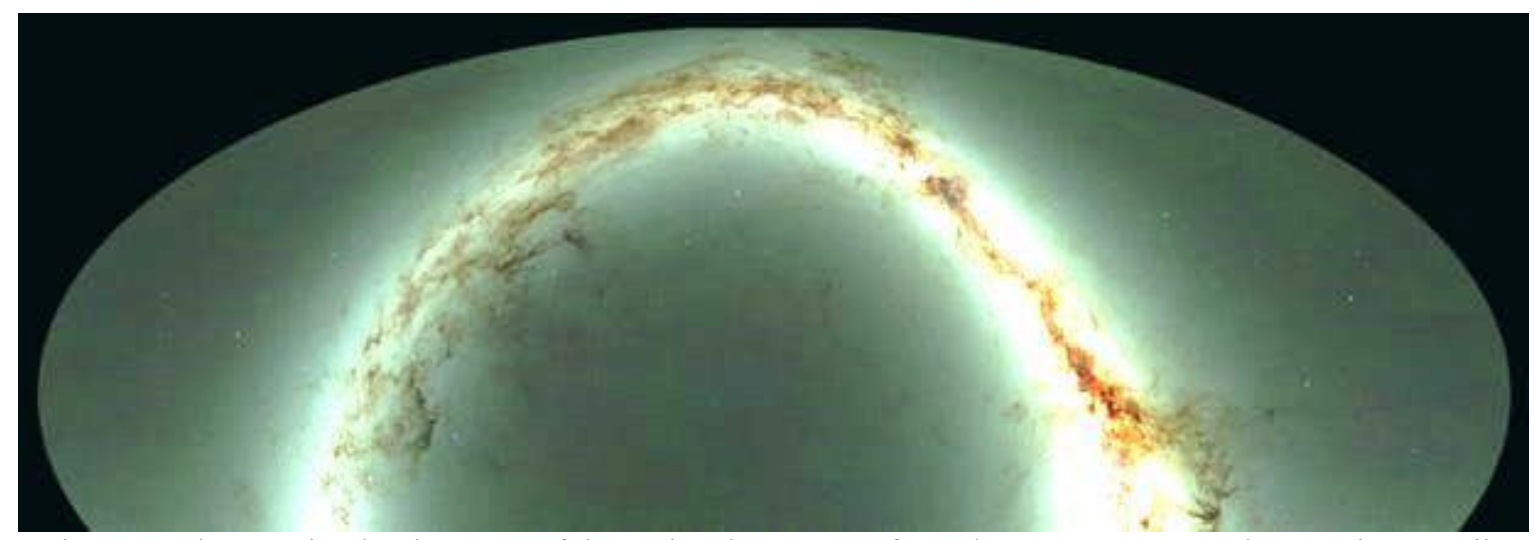

Figure 1. The "Static Sky, is a map of the entire sky, as seen from the Pan-STARRS telescope in Hawaii" (Credit: Danny Farro Science Consortium and Max Planck Institute for Extraterrestrial Physics).Posted by Newatlas.com (Color online) 


\subsubsection{The Cosmic Bosonic Vacuum Field}

The horizontal envelope is matter's waveform, it is the cosmic bosonic vacuum field which we call "Absolute Universe $\mathrm{U}_{\mathrm{w}}^{*}$ ", it accounts for many observational effects including: Newtonian gravitation, the strong nuclear force (SNF), electron magnetic moment, electric permittivity constant, magnetic permeability constant, electric polarizability, nuclear magnetic moment, radioactivity and metric expansion of space, Obande (2017a). The classical mass formula holds strictly only in this field where we have $\phi_{w}=h \vartheta_{w} / m_{w} c^{2}=1.0$, Obande (2015a).

\subsubsection{The Fermionic Field (Particulate matter) Universes}

The vertical envelope comprises three condensed matter fermionic fields, each is a universe including the invisible Component of the Absolute $\mathrm{U}_{\mathrm{p}}^{*}$, our Visible $\mathrm{U}_{\mathrm{p}}^{\mathrm{p}}$ and our invisible Analogue $\mathrm{U}_{\mathrm{p}}^{\prime}$; they are co-existent, exchange mass-energy m-e matrices and made up of clusters, nebulae, galaxies, stars, satellites of the stars, et cetera. These material universes are collectively responsible for such effects as gravitational acceleration, magnetic flux density, the fine structure constant, electron Compton wavelength and nuclear quadrupole moment. The classical mass formula fails to apply strictly here as we find $\phi_{p}=h_{p}^{x} / h \vartheta_{p}^{x} c^{o 2}=k$ where $\mathrm{x}$ indicates the universe and all three identify with the common fermion transverse field $c^{o}=3.71535229 \times 10^{-14} \mathrm{~m} / \mathrm{s}$; the coefficient $\mathrm{k}$ is element and universe specific, its value 1.0172 obtains for most elements in $\mathrm{U}_{w}^{*}, \mathrm{U}_{\mathrm{p}}^{*}$ and $\mathrm{U}_{\mathrm{p}}^{\mathrm{o}}$ but fluctuates significantly in $\mathrm{U}_{\mathrm{p}}^{\prime}$ where it records the figures 10 to 102, the latter is recorded by Fe, Obande (2015a), it would account for Fe's exceptional binding energy.

\subsubsection{Relative Brightness of the two Envelopes}

Relative brightness of the two envelopes reflects atomic energy disparity between the boson (b) and fermion (f) fields, the energy ratio $\mathrm{E}_{\mathrm{f}} / \mathrm{E}_{\mathrm{b}}$ varies from 1.0 to 102 /atom as explained above. Avogadro number, i.e., $6.23 \times 10^{43}$, Obande (2015a), multiples of this difference amounts to an immense aggregate molar energy contribution from zillions of tons of material objects in the three particulate matter universes to the brightness of the fermionic envelope.

An attempt to brighten the downloaded New Atlas' image produced a surprise; the vertical envelope resolves into three bands of shades if the image is printed in black and white with reduced contrast (ink-deficient cartridge); we attribute these bands to the three particle generations, with diminishing order of brightness from the center we have $\mathrm{U}_{\mathrm{p}}^{\mathrm{o}} \gg \mathrm{U}_{\mathrm{p}}^{\prime}>\mathrm{U}_{\mathrm{p}}^{*}$. It is the first evidence that the normally invisible $\mathrm{U}_{\mathrm{p}}^{\prime}$ and $\mathrm{U}_{\mathrm{p}}^{*}$ do indeed give off measurable radiation if the procedure adopted by the Pan-STARRS scientists is followed, it points to an exciting possibility for "dark matter" research.

\subsubsection{Orthogonality of the Fermion Field Universes and Invisibility of $U_{p}^{*}$ and $U_{p}^{\prime}$}

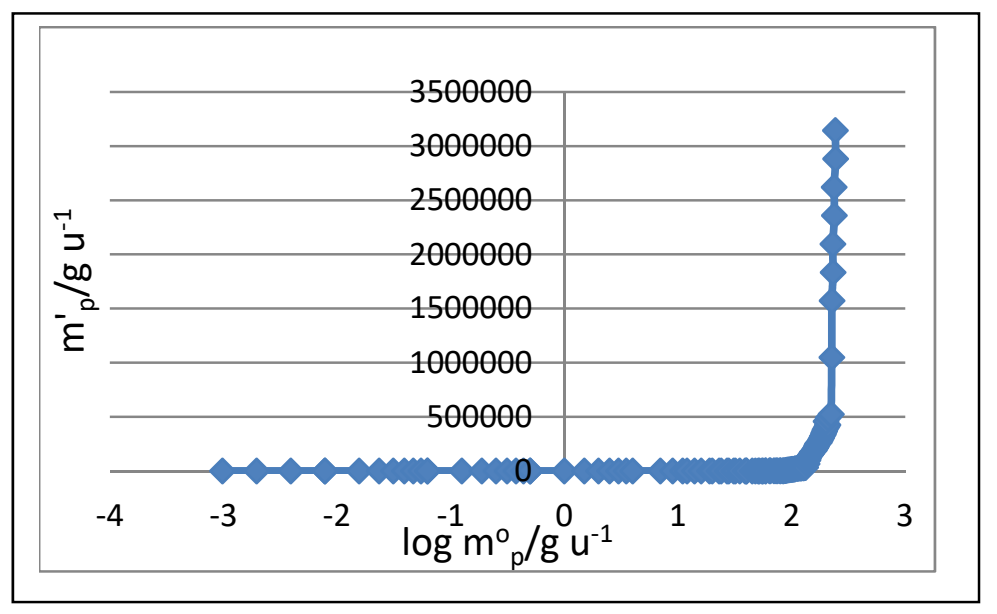

Figure 2. Plot of $\mathrm{m}_{\mathrm{p}}^{\prime}$ vs. $\log \mathrm{m}_{\mathrm{p}}^{\mathrm{o}}$

Figure 2 is a correlation of $\mathrm{m}_{\mathrm{p}}^{\prime} / \mathrm{u}$ vs. $\log \mathrm{m}_{\mathrm{p}}^{\mathrm{o}} / \mathrm{u}$, use of logarithmic scale for $\mathrm{m}_{\mathrm{p}}^{\mathrm{o}}$ merely facilitates a smooth rather than staggered curve. The figure reveals that m-e matrices of the two universes are orthogonal, and indeed all three particle-generation universes are mutually orthogonal; the subject is a bit involved, it traces to radioactivity and includes topics that fall outside the scope of this report. We see no immediate reason to ascribe some special qualities (such as life forms and intelligent beings) to our visible universe $\mathrm{U}_{\mathrm{p}}^{\mathrm{o}}$ which nature would contrive to deny our invisible co-tenants $\mathrm{U}_{\mathrm{p}}^{*}$ or $\mathrm{U}_{\mathrm{p}}^{\prime}$ or, indeed, $\mathrm{U}_{\mathrm{w}}^{*}$, we expect, therefore, that same chemical reactions that produce visible matter and life forms in $\mathrm{U}_{\mathrm{p}}^{\mathrm{o}}$ would do likewise in the invisible. Invisibility has earned them the appropriate 
tag "dark matter/dark energy", however, their contributions to our observational physical parameters, strongly speak to their physical existence, Obande (2017a). We celebrate Figure 1 as it resolves so many pending issues in contemporary atomic physics. $U^{*}$ is a waveform, characteristically invisible, however, the question of invisibility of $U_{p}^{*}$ and $U_{p}^{\prime}$ remains pressing, we note as follows: i) existence of two or more material universes that co-exist with and exchange spacetime matrices with our universe is entirely new to physics, at the moment there is no theoretical framework for information (light) communication across mutually orthogonal ref. frames; ii) as evident in Figure 2, there is continuous matrix exchange between the three material universes, in the visible universe the exchange gives rise to high energy $\gamma$ and $\alpha$ radiations, Obande (2016b), particularly those associated with black holes and the cosmic large scale, Cartlidge (2011), Cowan (2011), Dylan (2014), Borghino (2015).

\subsection{Bifurcation of the Visible Universe}

Figure 3 is a plot of $\vartheta_{p}^{\mathrm{x}}$ vs. $Z_{\mathrm{n}}$ for $\mathrm{U}_{\mathrm{p}}^{*}, \mathrm{U}_{\mathrm{p}}^{\mathrm{o}}$ and $\mathrm{U}_{\mathrm{p}}^{\prime}$, where $Z_{\mathrm{n}}$ is the element's atomic number in nature's chemical periodicity, Obande (2016a). Notice the bulge in $\vartheta_{\mathrm{p}}^{\prime}$ values at the $5^{\text {th }}$ chemical period/space octave, the interval $Z_{\mathrm{n}}$ $=30$ to 35 , and observe that beyond this interval values of $\vartheta_{\mathrm{p}}^{\circ}$ degrade progressively relative to corresponding $\vartheta_{\mathrm{p}}^{*}$ and $\vartheta^{\prime}$ p values down to the end of the periodicity, it presents a bifurcated visible universe. The feature makes a lengthy subject which again cannot be taken here; briefly, i) it is responsible for proximate linear atomic mass growth rate in $\mathrm{U}^{\circ}{ }_{\mathrm{p}}$ relative to logarithmic growth rates in $\mathrm{U}^{*}{ }_{\mathrm{w}}, \mathrm{U}_{\mathrm{p}}^{*}$ and $\mathrm{U}_{\mathrm{p}}^{\prime}$; ii) it marks the periodic space wherein our invisible analogue $U_{p}^{\prime}$ exchanges spacetime matrices with the visible $U^{\circ}$; iii) it traces to the classical black hole; a detailed report on the subject is in progress.

The quantitative implication of the bifurcation is remarkable. It divides the visible universe into three distinct blocks of chemical elements: i) the linear mass progression from electron e to $\mathrm{Na}$ which includes 22 inaccessible elements existing between e and H conventionally called "elementary particles", Obande (2016a); ii) the inflexion block from Li to Mg, it gives rise to the bulge in Figure 3 and associates with the classical black hole, and iii) the pseudo-logarithmic rest mass growth rate from $\mathrm{Mg}$ to the end of the periodicity. Our investigation reveals that the interval $Z_{n}=30-35$ marks spacetime quanta that demarcate material and spatial periodicities, it is nature's device for $m$-e matrix exchange between $U_{p}{ }_{p}$ and $U_{p}^{\prime}$, relative atomic mass $m_{r}$ values in our universe owe to this device, i.e., $\mathrm{m}_{\mathrm{r}}\left(\mathrm{m}_{\mathrm{p}}^{\mathrm{o}}\right)=\mathrm{m}_{\mathrm{p}}^{*} \pm \mathrm{m}_{\mathrm{p}}^{\prime}$; before the device, the sum applies and after it the difference applies. An attempt to rid the simulation of reference to empirical $\mathrm{m}_{\mathrm{r}}$ values produced the expression to $\mathrm{m}^{\mathrm{o}} \mathrm{p}=\vartheta_{\mathrm{p}}^{\prime}(1 \pm \psi) / 2048$, where the ratiot $\psi=\vartheta_{\mathrm{p}}^{\prime} / \vartheta_{\mathrm{p}}^{*}$ and $\vartheta^{*}{ }_{\mathrm{w}(\mathrm{H})}=\vartheta_{\mathrm{p}(\mathrm{H})}^{*}=\vartheta_{\mathrm{p}(\mathrm{H})}^{\prime}=2048$; it shows that $\mathrm{U}_{\mathrm{p}} \mathrm{o}_{\mathrm{p}}$ results from non-linear wave dynamics of $U_{p}^{*}$ and $U_{p}^{\prime}$.

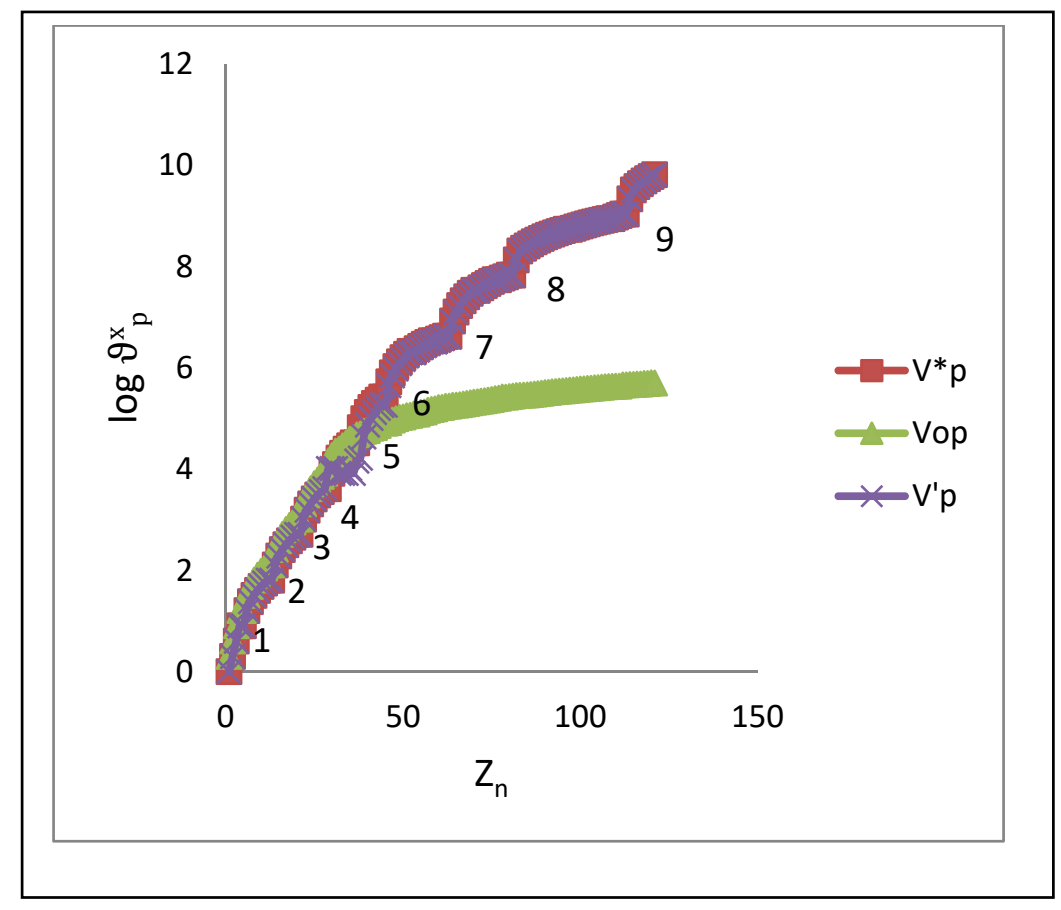

Figure 3. Plot of $\log \vartheta^{\mathrm{x}} \mathrm{v}$ vs. $Z_{\mathrm{n}}$ (Color online) 


\subsection{Radioactivity, Stellar Explosion and Metric Space Expansion}

\subsubsection{Radioactivity and Stellar Explosion/Supernova}

Conventional notion attributes radioactivity to instability of the atomic nucleus with nuclidic content as culprit. According to this notion, the atom achieves stability through energy loss in the form of $\alpha-, \beta$ - and neutrino decays, $\gamma$-ray emission and/or e-capture. Radioactivity is believed to be a random ("stochastic") process, impossible to predict regardless of how long the atom existed. Three models are adjudged successful to partially or fully account for the effect: the collective model; independent particle model; and the combined model, acclaimed most successful of the three, Halliday et al. (2002). Beyond numerical nuclidic proton-neutron balance, particularly the concept of magic numbers, conventional notion sees no further details on the specific causality of nuclear instability.

Classical Newtonian physics, however, traces radioactivity to convergence of absolute values of tensile properties of the waveform e-m harmonic oscillator, it reveals that spontaneous radioactivity occurs once the oscillator's angular speed attains a critical value, Obande (2015b). A recent investigation of the familiar physical constant $|\mathrm{e}-|=1.6022 \times 10^{-19}$ yields the relationship,

$$
\rho_{w} \vartheta_{w}^{4}=3 m_{w} / \omega_{w} c^{3}=1.0691 \times 10^{-19} \mathrm{~N} \mathrm{~m} /(\mathrm{m} / \mathrm{s})^{3} \mathrm{rrd}^{-1} \mathrm{~s}^{-1}
$$

Eq. (1) describes "an effect in which the torque field imposes an accelerated (bulk) compression which couples with angular speed to impact an aggressive hydrostatic pressure on the bosonic envelope", Obande (2017a). It is further posited that: i) on atomic scale, precisely at $\mathrm{At}\left(\mathrm{m}_{\mathrm{r}}=0.210 \mathrm{~kg} / \mathrm{u}, \omega^{\mathrm{o}} \mathrm{p}=2 \times 10^{10} \mathrm{rad} \mathrm{s}^{-1}\right)$, the effect initiates spontaneous radioactivity, and ii) on stellar and galactic scales, it is responsible for stellar explosion/supernova at a yet to be investigated $\omega$ value. Thus, classical analysis reveals that variation of vacuum radiation density with oscillation frequency gives rise to spontaneous radioactivity at a critical angular speed value; notably, the same parametric combination initiates radioactivity on atomic scale and stellar explosion on cosmological scale. In other words, stellar explosion/supernova is cosmological equivalent of spontaneous radioactivity, it adds an important cosmological dimension to universal discrete scale relativity.

Figs. 4 and 5 illustrate causality of spontaneous radioactivity with plots of $\tau^{*}{ }_{\mathrm{w}}$ vs. $\omega^{*}{ }_{\mathrm{w}} / \mathrm{r}_{\mathrm{w}}{ }_{\mathrm{w}}$ and $\tau_{\mathrm{w}}{ }_{\mathrm{w}}$ vs. $Z_{\mathrm{n}}$ respectively. Observe as follows: i) radioactivity is caused exclusively by changes in tensile properties of the boson field that encases fermionic matter exactly as shown in Figure 1; in other words, the wave component of the atom exercises significant control over the particulate matter component likewise, the cosmic vacuum field exercises similar control over its material cosmological content; ii) Figure 4 pinpoints spontaneous radioactivity at At, $\tau^{*}{ }_{\mathrm{w}}=$ 2.14, i.e., 214\%; iii) Figure 5 facilitates visualization of the stepwise process of nuclear instability that leads to radioactivity. Discernible evidence of instability commences at $\mathrm{Ru}, \mathrm{Z}_{\mathrm{n}}=70$, where a change becomes noticeable in the $\tau^{*}{ }_{\mathrm{w}} / Z_{\mathrm{n}}$ gradient, the change progresses gradually up to $\mathrm{Xe}, Z_{\mathrm{n}}=80$ where a sudden jump occurs signifying considerable instability albeit insufficient to initiate spontaneity which eventually occurs at $A t, Z_{n}=111$, Obande (2015c). The quotient $\tau /(\omega / \mathrm{r})$ denotes electrostatics effect, it manifests atomic mass, Obande (2016a), it would account for m-e packet ejection at spontaneity as reflected in the staggered mass distribution. Given that 1.0 mole contains $6.23 \times 10^{43}$ (not $6.02 \times 10^{23}$ ) particles, even if only a few m-e packets are ejected per second, it would account for values of half lives of most natural radioactive decays.

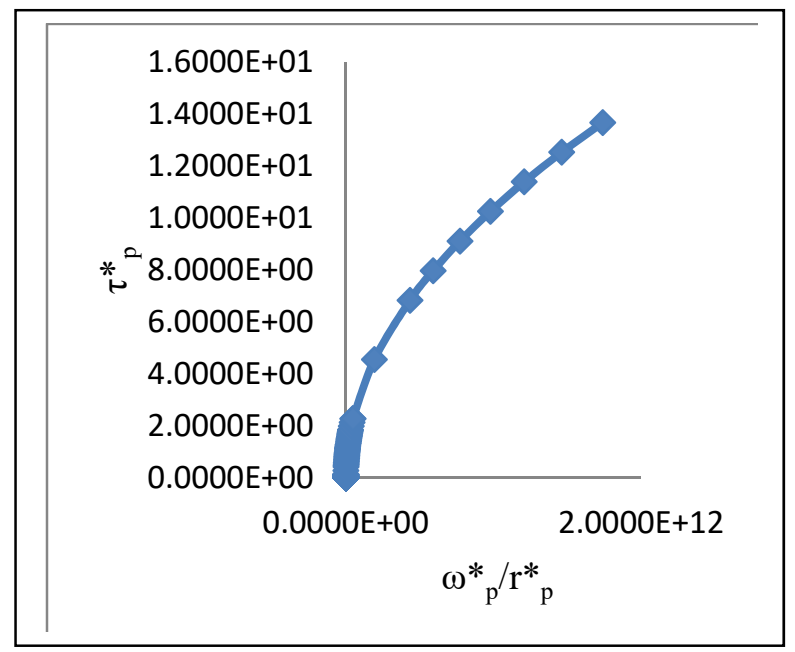

Figure 4. Plot of $\tau^{*}{ }_{\mathrm{w}}$ vs. $\omega^{*}{ }_{\mathrm{w}} / \mathrm{r}_{\mathrm{w}}{ }_{\mathrm{w}}$

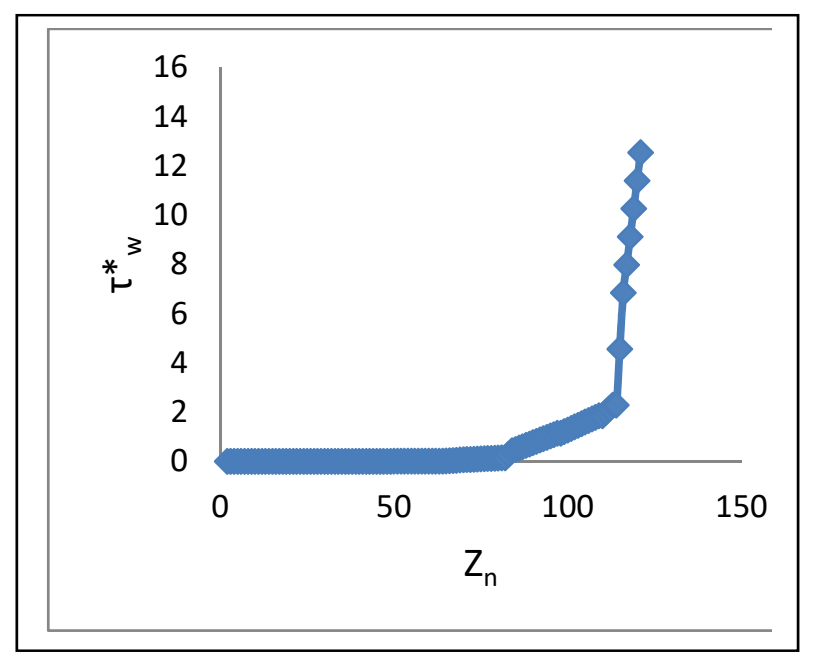

Figure 5. Plot of $\tau_{\mathrm{w}}^{*}$ vs. $Z_{\mathrm{n}}$ 


\subsubsection{Metric Expansion of Space}

Few issues command wider physical research attention than the nature and causality of space expansion yet, several questions remain pressing. Is space actually expanding or is it a classical de Sitter (1917) static universe? If expanding, is it Hubble's (1929) radial motion as formalized by Hoyle (1948), or is it angular motion as some insist?, Peebles \& Ratra (2003), Longo (2011), Raghuprasad (2013), Jakubowski (2016).

In a tabulation titled "Timeline of Cosmology" Wikipedia provides a succinct but quite informative highlight on development of cosmological theories from Hindu Rigveda (2000BC) to the reigning paradigm. Notably, the table provides snapshots of the cosmologies of Einstein, de Sitter, Friedmann, Lemaitre (Big Bang Model, BBM), Milne \& McCrea, Robertson \& Walker, Bondi, Klein, Guth, Steinhardt \& Turok, and Baum \& Frampton. It highlights an epic marked with key developments, notably, Friedmann's (1922) field equation indicating expanding space; Hubble's (1929) observational evidence, and Lemaitre's (1931) construction of the BBM. From Einstein's (1920) original static finite universe, modern Standard Cosmological Model SCM uses the Hubble Constant to construct first, a linearly expanding space, Hoyle (1948), then an accelerating universe, Reiss (1998), Floerchinger (2015) and now, recent observational evidence would seem to favor a return to a linear but incredibly fast expansion, Nielsen et al. (2016), Reiss et al. (2016), Castelvecchi (2016).

Notably, majority positions on space expansion derive from redshift measurement. In line with sound wave Doppler shift, it would seem quite natural to interpret Hubble's (1929) results in terms of radial motion; the same notion had earlier informed Slipher's (1913) interpretation of radial velocity of Andromeda Nebula. It is, therefore, a matter-of-course for same notion to inform Lemaitre's (1931) BBM and Hoyle's (1948) expanding universe model. The question of whether or not cosmological redshift is due to radial or angular motion remains open to date. One thing is certain, most cosmological structures literally spin on their axis, however, the effect is not quantitatively explicit at the quantum level.

Quantum mechanics QM notion of spin replaces Uhlenbeck and Goudsmit's (1926) explicit definition with a nonspecific notion that differs significantly from literal physical rotation; for instance, Wikipedia says, "In 1925, Ralph Kronig, George Uhlenbeck and Samuel Goudsmit at Leiden University suggested an erroneous physical interpretation of particles spinning on their own axis", emphasis ours. Thus, spin in QM admits of quantized (classical) angular momentum but rejects (quantized) classical physical rotation, it makes it a bit challenging to rationalize QM's spin with observational evidence see, for instance, Moskowitz (2014) and Siegel (2017). One would have expected that by now there should be no doubt regarding universality of scale-free spin in the context of physical rotation; it comes quite naturally in analysis of the classical quantum state, Obande $(2015 \mathrm{~b}, 2015 \mathrm{c}$, 2016a) and in observational cosmology, Rubin (2000). Indeed, existence as we know it in terms of say, gravitation, magnetism and random (thermal) motion would cease without scale-free physical rotation of matter waves, Obande (2017a). We see no peculiar characteristics capable of precluding the atom or "sub-atomic" particle from physical rotation. The situation is traceable to statistical mechanics, the subject contributed much to early development of QM, e.g., Pauli (1940), if the development had been informed by the atomic quantum e-m harmonic oscillator, physics might have taken an entirely different course, likely a lot more observational, conceptual and cognitive than the reigning paradigm.

Classical analysis reveals that space is indeed not accelerating but "expanding" at a superluminal velocity; the parametric interaction causality is spatial variation of bosonic flux density with stress field, interestingly, it identifies also with the ubiquitous $|\mathrm{e}-|=1.6022 \times 10^{-19}$, Obande (2017a); a log-log plot of $\rho_{\mathrm{w}}$ vs. $\sigma_{\mathrm{w}}$ yields,

$$
\rho_{w} / \sigma_{w}=8.511410^{-19}(\mathrm{~m} \mathrm{rad} / \mathrm{s})^{-2}
$$

Dimensional analysis gives $\rho_{w} / \sigma_{w}=3 / 4\left(r_{w} \omega_{w}\right)^{2}=3.381910^{-18}(\mathrm{~m} / \mathrm{s})^{-2}$, i.e., $\mathrm{v}_{\mathrm{W}}=4.709 \times 10^{8} \mathrm{~m} / \mathrm{s}$; in plain words, the vacuum field is moving tangentially at superluminal velocity $1.57 \mathrm{c} \mathrm{m} / \mathrm{s}$. We hasten to note here that the denominator 4 was omitted from the coefficient $3 / 4$ in our earlier report, Obande (2017a), it gave the wrong value $\mathrm{V}_{\mathrm{W}}=\pi \mathrm{c}$, instead of $\sim 0.5 \pi \mathrm{c}$; the final conclusion that vacuum space is moving tangentially at superluminal velocity is not affected but the correct value of the velocity field is about half of the reported value, the error is regretted. Given the vacuum density $\rho_{\mathrm{w}}=2.609 \times 10^{-36} \mathrm{~kg} / \mathrm{m}^{3}$, Obande (2016b) and stress field $\sigma_{\mathrm{w}}=3.086 \times 10^{-18} \mathrm{~Pa}$, Obande (2015b), theory suggests that the rate of metric space expansion is assessable from tensile properties of the vacuum.

Notably, the result $(\mathrm{v}=\mathrm{r} \omega)$ shows clearly that space is not expanding radially, the effect is tangential velocity; more significantly, spatial expansion is not caused by negative gravitation, cosmological constant or "dark energy", it results from an ingenious field engineering whereby the field density/stress quotient produces a constant superluminal tangential velocity field, i.e., isotropic, isostasic, bolometric vacuum space subjected to a constant hydrostatic shear stress complies through a constant superluminal angular speed. 


\subsection{The Static Sky and Universal Conformal Invariance}

For the very first time in history, mankind is able to see the primitive structure of reality, by all standards it is the greatest empirical feat since Galileo. Figure 1 is an image of the primitive structure of every material object including the isolated atom, "sub-atomic" particle, molecule, cell, organism, animate and inanimate forms and, of course, the cosmos. The literature on discrete scale relativity such as Oldershaw $(2007,2014)$, Fedosin (2017) and Wikiversity, might seem to lack direct bearing to Figure 1 and that informs our goal in this paper - to show that the "Static Sky" makes the most powerful addition to existing evidences that conformal invariance is a fundamental attribute of nature. From the atom to the cosmos, matter intrinsically comprises wave and particulate forms orthogonally positioned as shown in Figure 1; it informs observationals like relative orthogonality of major axes of the developing chick and the egg shell or the mammalian embryo and the womb, its presence at the level of the cell has been identified with cosmological structures, Oldershaw (2014), Berry et al. (2016). Without prior knowledge, our recent classical analysis produced evidence that took us by surprise and led to a literature search that called our attention to the subject, Obande (2017b). Right from the onset, our investigations have consistently compelled definition of reality with a clear distinction between the atomic wave and particulate forms that transform to the cosmic large scale comprising the vacuum (boson) field and condensed matter (fermion) field, it informs our sheer excitement with the visible evidence.

So, how does evidence for wave-particle nature of reality answer all existing and conceivable questions in physics? There is no short answer to that question; the implications are enormous and we must expect that every atomic physicist is well aware of the fact. Simply, it calls for a major review of the conventional fundamental approach. Hitherto, physics has focused on only one half of reality, now we have observational evidence that presents the complete picture; it merges cosmology, astrophysics and atomic physics into a single profound physical discipline that identifies with same classical Newtonian physics which differentiates only in values of extensive properties. It suddenly promotes the telescope a most invaluable physical research tool on equal, if not superior, footing with the accelerator for investigating internal structure and properties of the isolated atom.

This is a summary of our take on Figure 1: discrete scale relativity informs that the cluster and galactic dimensional hierarchicals replicate chemical periodicity in which the galaxy is equivalent to the chemical element. The galaxy in turn comprises a periodicity in which the star is equivalent to the chemical element and the star comprises a periodicity in which the satellite (planet) is equivalent to the element; thus, an investigation of cosmological dynamics corresponds to an investigation of atomic dynamics, the two extreme scales identify with same formalism but differ only in extensive properties.

\subsection{Size of the Cosmos and the Big Bang Model}

\subsubsection{Size of the Universe}

Until some theoretician (often mathematician) comes up with (usually compelling) quantitative accounts of why what we see might not be what we think, thanks to Figure 1, we now have a visible evidence to address whether or not the observable cosmos is (visually not mathematically) flat or curved, open or closed, finite or infinite. We must await the outcome of detailed evaluation of the image's spatial dimensions to get an idea of its total size and, possibly, dimensions of the constituent envelopes, i.e., the universes $U_{p}^{*}, U_{p}^{o}$ and $U_{p}^{\prime}$. However, the question of its boundary, and by implication exact shape, might not be as simple as the image would suggest; for certain, the boundary would be an impenetrable invisible pitch-black zero-Kelvin vacuum, the shape of the entire system would depend on its symmetry group. We have argued that every natural form, be it electron or the galaxy, belongs to one of only four symmetry groups, Obande (2017b); it invariably starts off as symmetry group SG 1, ideally a perfect sphere, or cycle in 2D, matures as SG 4, ideally a perfect cube or sphere and evaporates into space through the tortuous gradual processes of highly distorted SG 3, SG 2 and finally, again SG 1, see the image posted by Sparknotes (2015) of an evaporating cosmic object in highly distorted, indeed fragmented, SG 1. If the two elliptical envelopes of Figure 1 were re-constructed and the vertices joined, we would get a rectangle which ideally would be a square for SG 4 in 2D, a deviation from the square would indicate corresponding deviation from full maturity and gradual slide into distortion. Given the previous and present analyses, we take the position that Figure 1 is the current image of our observable cosmos, it would seem to suggest a healthy state of maturity for our cosmos; but cosmology attests to the fact that the state of health of the cosmic envelope does not necessarily translate to the state of health of constituent galaxies; it should be possible to assess the state of health of a galaxy, and possibly a stellar system, when the subject is sufficiently developed. Majority literature estimates put the diameter of the visible cosmos at $90( \pm 2)$ billion light years, Carlstrom (2015), Azher (2016) and Redd (2017); however, as observed by NASA $(2015,2017)$, at the moment no one knows for certain the size of the whole universe. 


\subsubsection{The Big Bang Model}

Two key observational evidences claim support for the BBM: the Hubble Constant $\mathrm{HC}$ and the Cosmic Microwave Background $\mathrm{CMB}$. We find above that $\mathrm{HC}$ refers to angular not radial motion, space is not expanding into newly created matrices, it identifies with varied scales of angular motion; we examine the case for the CMB.

Contrary to literature, Hu et al. (1997), The Task Force Report (2005), Bennet (2006), Bennet et al. (2013), Peplow (2013), the CMB is not an "after glow" of BB's original radiation, it is the vacuum field, i.e., classical zero-point radiation. Planck energy $h \vartheta$ of the chemical elements' waveforms vary from electron's $\mathrm{E}_{\mathrm{e}(\mathrm{w})}=6.6261 \times 10^{-34} \mathrm{~J} /$ atom to americium's $\mathrm{E}_{\mathrm{Am}(\mathrm{w})}=4.2688 \times 10^{-24} \mathrm{~J} /$ atom, Obande (2015a). A vacuum property obtains not as an average but summation of each element E's value, Obande (2016c) thus, we have $\sum \mathrm{E}_{\mathrm{w}(\mathrm{E})}=3.6749 \times 10^{-23} \mathrm{~J}$; it gives (delete space) $\mathrm{T}=\sum \mathrm{E}_{\mathrm{w}} / \mathrm{k}=3.6749 \times 10^{-23} / 1.381 \times 10^{-23}=2.662 \mathrm{~K}$, a value well in line with empirical $2.725 \mathrm{~K}$, Big Bang (2017). We must call attention to wrong attribution of the CMB to de Broglie radiation in all preceding reports, the error arose from avoidable inadequate attention to details whenever the subject is not the principal object of investigation, it is regretted. The present analysis immediately accounts for anisotropy. The CMB arises from only bosonic envelopes that enclose fermionic packets as shown in Figure 1, it demarcates concentrations of matter, with zero Kelvin voids in between envelopes as shown in the WAMP map, Bennet (2013); compare, for instance, the "... WAMP image..." and the "Panoramic view of the entire infra-red sky..." in (Big Bang 2017), juxtaposition of transparencies of the two images would make interesting comparison.

Some respectable opinions have persistently refuted existence of a link between the CMB and the BB, Assis and Neves (1995), Arp (2005), Mersini-Houghton (2014); Figure 1 is, perhaps, the best large-scale visual evidence yet revealing a profoundly ordered cosmic envelope. Now that we have a visual evidence, some might want to work out a quantitative procedure that permits an uncontrolled cataclysmic cosmic explosion to eventually cool to the ordered system of Figure 1 but, certainly it would be taking an expensive joke much too far to attempt an account of the separation and mutual orthogonal orientation of the boson and fermion fields. Already, an attempt exists that explains away discrete scale relativity in the context of a "... Background of the Cosmological Collider", Chen et al. (2017); in other words, physics is rapidly losing its science to a veritable mathematical gymnastics.

\section{Summary and Conclusion}

i. Image of the entire sky, the "Static Sky" recently released to the public by scientists at the PanSTARRS1 telescope in Hawaii is analysed. It depicts, for the very first time, nature's intrinsic waveparticle duality.

ii. The horizontal fine-grained darker envelope is nature's waveform, i.e., the imponderable bosonic cosmic vacuum field, while the brighter coarser-grained vertical envelope belongs to composite radiation of visible and invisible particulate matter fields. Relative brightness of the two envelopes reflects quantifiable energy difference between the atomic wave and particulate forms; mass-energy $\mathrm{m}$-e equivalence holds strictly only in the vacuum field where the wave/particle energy coefficient is unity, i.e., $\phi_{\mathrm{w}}=\mathrm{h} \vartheta / \mathrm{mc}^{2}=1.0$, the same coefficient is greater than unity in the fermionic matter field, i.e., $\phi_{\mathrm{p}}=\mathrm{h} \vartheta_{\mathrm{p}}^{\mathrm{x}} / \mathrm{m}_{\mathrm{p}}^{\mathrm{x}} \mathrm{c}^{22}=\mathrm{k}$, where $\mathrm{k}$ varies in value from 1.0172 to 102 , index $\mathrm{x}$ refers to the ref. frame and $\mathrm{c}^{\mathrm{o}}$ is invariant transverse field of particulate matter radiation.

iii. Earlier investigations were cited to inform that corresponding bosonic field envelope encloses every particulate matter exactly as shown in the Static Sky image, it is exclusively responsible for observational effects such as gravitation, radioactivity, metric space expansion, vacuum permittivity, magnetic permeability and the strong nuclear force that holds matter together on all, from submicroscopic to cosmic, scales.

iv. Earlier investigations were also cited to inform that the vertical coarse-grained brighter envelope comprises three ref. frames or universes with common chemical periodicity, two invisible material universes co-exist in harmony with our visible universe, all three are mutually perpendicular. Particulate matter (fermionic) field exclusively manifests the fine structure constant, magnetic flux density, electron Compton wavelength and gravitational acceleration.

v. The visible universe is bifurcated and the effect is responsible for its proximate linear atomic mass growth rate as against logarithmic growth rates in the invisible universes; the bifurcation occurs within a narrow atomic mass interval which marks periodic space within which the visible universe and its invisible analogue exchange m-e matrices.

vi. The two main observational evidences supporting the Big Bang model are faulted on fundamental theoretical grounds which show that: a) cosmological redshift phenomena arise from axial rather than radial motion, the effect is manifested by the vacuum field parametric self-interaction $\rho_{\mathrm{w}} / \sigma_{\mathrm{w}}=8.5114$ $\mathrm{x} 10^{-19}(\mathrm{~m} \mathrm{rad} / \mathrm{s})^{-2}$ whose dimensional analysis yields the superluminal angular velocity $\mathrm{v}_{\mathrm{w}}=4.709 \mathrm{x}$ $10^{8} \mathrm{~m} / \mathrm{s} ; \mathrm{b}$ ) the cosmic microwave background CMB radiation is classical zero-point temperature easily 
evaluated from summation of bosonic transverse fields' energies $E_{\mathrm{w}}=h \vartheta_{\mathrm{w}}$ of the chemical elements, $\mathrm{T}=\sum_{e}^{A m} E_{w} / \mathrm{k}=3.675 \times 10^{-23} \mathrm{~J} / 1.38110^{-23} \mathrm{~J} / \mathrm{K}=1.662 \mathrm{~K}$.

We conclude that the Pan-STARRS" "Static Sky" is indeed physics all-time goldmine whose theoretical and empirical potentials are likely inexhaustible in the foreseeable future. Given the mounting evidences for universality of discrete scale relativity DSR, the Static Sky merges atomic physics with astrophysics and cosmology in a way never before imaginable. We expect that when fully developed DSR, with the assistance of topology, would subsume all science disciples in physics.

\section{Recommendation}

In our opinion, since Galileo, there has been no greater empirical contribution to physical knowledge of reality than the "Static Sky". Without considering ourselves qualified to do so, we crave indulgence to recommend to the physical community for the highest decoration in physics, the key contributor(s), without whom realization of Figure 1 would be either impossible or of much lower definition for meaningful analysis.

\section{References}

Arp, H. (2005). Observational cosmology: from high redshift galaxies to the blue pacific. Progress in Physics, 3, 3.

Assis, A. K., \& Neves, M. C. (1995). History of the 2.7 K temperature prior to Penzias and Wilson. Apeiron, 2(3), 79-87.

Azher, A. (2016). What is the size of the universe in cm? Retrieved from https://socratic.org/questions/what-is-thesize-of-the-universe-in-centimeters

Bennett, C. L. (2006). Cosmology from start to finish. Nature, 440(7088), 1126-1131. http://dx.doi.org/10.1038/ nature 04803

Bennett, C. L., Larson, D., Weiland, J. L., Jarosik, N., Hinshaw, G., Odegard, N., ... \& Komatsu, E. (2013). Nineyear Wilkinson Microwave Anisotropy Probe (WMAP) observations: final maps and results. The Astrophysical Journal Supplement Series, 208(2), 20. http://arxiv.org/abs/1212.5225

Berry, D. K., Caplan, M. E., Horowitz, C. J., Huber, G., \& Schneider, A. S. (2016). "Parking-garage" structures in nuclear astrophysics and cellular biophysics. Physical Review C, 94(5), 055801. https://dx.doi.org10.1103PhysRevC.94.55801

Big Bang. (2017). In Wikipedia, the free encyclopedia. Retrieved from http://en.wikipedia.org/wiki/BigBang

Borghino, D. (2015). New model suggests dark matter is made up of electrically charged tparticles. Gizmag magazine Sept. 27.

Carlstrom, J. E., Crawford, T. M., \& Knox, L. (2015). Particle physics and the cosmic microwave background. Physics Today, 68(3), 28-34. Retrieved from

http://scitation.aip.org/content/aip/magazine/ physicstoday/article/68/3

Cartlidge, E. (2012). Gamma rays hint at Dark Matter. Retrieved from http://physicsworld.com/cws/article/ news/2012/apr/24/gamma-rays-hint-at-dark-matter

Castelvecchi, D. (2016). Measurment of Universe's expansion rate creates cosmological puzzle. Nature News \& Comments 14 ${ }^{\text {th }}$ April. Retrieved from https://www.nature.com/news/measurement-of-univessesexpansion-rate-creates-cosmological-puzzle-1.19715?WT.mc_id=SFB_NNEWS_1508_RHBox

Chen, X., Wang, Y., \& Xianyu, Z. Z. (2017). Standard Model background of the cosmological collider. Physical Review Letters, 118(26), 261302. https://dx.doi.org10.1103/PhysRevLett116261302

Cowan, R. (2011). Shedding light on the mystery of dark matter. Nature News. http://dx.doi.org/10.1038/news. 2011.531

Einstein, A. (1920). Relativity: The Special and General Theory, trans. Robert W. Lawson (New York: Crown, 1961), 26. Retrieved from http://www.archive.org/details/cu392401180

Fedosin, S. (2017). Infinite Hierarchical Nesting of Matter. Retrieved from https://en.wikiversity.org./wiki/Essays/Fedosin/Infinite_hierarchical_Nesting_of_Matter

Floerchinger, S., Tetradis, N., \& Wiedemann, U. A. (2015). Accelerating cosmological expansion from shear and bulk viscosity. Physical review letters, 114(9), 091301. https://dx.doi.org/10.1103/PhysRevLett.091301

Francis, M. R. (2015). The Mystery of particle generations. Retrieved from http://www.symmetrymagazine.org/ article/august2015/the-mystery-of-particle-generations 
Friedman, A. A. (1922). Uber die Krummung des Raumes. Zeitschrift fur Physik, 10(1), 377.

Friedman, A. A. (1999). On Curvature of Space. Gen. Rel. Grav., 31(12), 1991 http://dx.doi.org/10.1023/A:10267 1225741

Gott III, J. R. (2005). A Map of the Universe. Astrophys. J., 624, 463 http://arxiv/abs/atsro-ph/0310571

Halliday, D., Resnick, R., \& Walker, J. (2002). Fundamentals of Physics. N.Y.: John Wiley.

Hoyle, F. (1948). A New Model for the Expanding Universe. Mont. Not. Roy. Astron. Soc., 108, 372. Retrieved from http://adsabs.havard.edu/abs/1948MNRAS.108..372H

Hu, W., Sugiyama, N., \& Silk, J. (1997). The physics of microwave background anisotropies. Nature, 386, 37.

Hubble, E. (1929). A relation between distance and radial velocity among extra-galactic nebulae. Proceedings of the National Academy of Sciences, 15(3), 168-173. http://dx.doi.org/10.1073/pnas/15.3.168

Jakubowski, P. (2016). Consiquences of the unification in physics II, Cosmic hierarchy of the solar system. Physics Essays, 29(1), 129. http://dx.doi.og/0836-1319-29.1.129

Lemaitre, G. (1931). The Evolution of the Universe: Discussion. Nature, 128(3234), 699.

Linden, T. (2014, August). The Characterization of the Gamma-Ray Signal from the Central Milky Way: A Compelling Case for Annihilating Dark Matter. In AAS/High Energy Astrophysics Division (Vol. 14). http://arxiv.org/abs/astro-ph/1402.6703

Longo, M. J. (2011). Circumferential movement rather than radial. Phys. Lett., G699(4), 244.

Mersini-Houghton, L. (2014). Black holes don't exist and Big Bang theory is wrong. Retrieved from http://www.huffington.com./2014/09/29/black-holes-dont-exist-n-5885940

Moskowitz, C. (2014). Proton Spin Mystery Gains a New Clue. Retrieved from http://www.scientificamerican.org/ article/proton-spin-mystery-mystery-gains-a...

NASA. (2015). The Cosmic Distance Scale. Retrieved from https://imagine.gsfc.nasa.gov/cosmic/farthest info.html

NASA. (2017). How big is our Universe? Retrieved from https://www.nasa.gov/audience/foreducators/58/F_How_Big_is_Our_Universe.html

National Science Foundation (USA). (2005). Report of the Task Force, Cosmic Microwave Background Research. Retrieved from http://www.nsf.gov/mps/ast/tfcr.jsp>(2005)

New Atlas. (2016). Static Universe. Retrieved from http://newatlas.com//pan-starrs-largest-digital-survey-visibleuniverse

Nielsen, J. T., Guffanti, A., \& Sakar, S. (2016). Marginal evidence for cosmic acceleration from Type 1A supernovae. Sci. Reports, 6, 35596. https://dx.doi.org/10.1038/sreo35596

Obande, O. P. (2013). Notes on Russellian cosmogony - Part I: Absolute Atomic Mass. Int. J. Eng. Sci., 2(4), 68. Retrieved from http://wwwtheijes.com/papers/v2-i4/part.\%20(2)/M0242058077.pdf

Obande, O. P. (2015a). Notes on Russellian Cosmogony. II.A procedure for theoretical evaluation of relative atomic mass and internal energy. Phys. Essays., 28(1). http://dx.doi.org/10.4006/0836-1398-28.1.78

Obande, O. P. (2015b). Classical mechanical analysis of the atomic wave and particulate forms. Int. J. Eng. Sci., 4(6), 1. Retrieved from http://www.theijes.com/papers/v4-i6/version-2/a046201011.pdf

Obande, O. P. (2015c). Classical Defifitions of Gravitation, Elelctricity and Magnetism. Appl. Phys. Res., 7(6), 85 http://dx.doi.org/10.5539/apr.v7n6p85

Obande, O. P. (2016a). Atomic mass: Origin, Units and Constants. Appl. Phys. Res., 8(1), 92. http://dx.doi.org/10. 5539/apr.v8n1p92

Obande, O. P. (2016b). On the Photon's Identity: Implications for Relativity and Cosmology. Appl. Phys. Res., 8(5), 10. http://dx.doi.org/10.5539/apr.v8n5p10

Obande, O. P. (2016c). A classical perspective of the cosmological constant. Phys. Essays., 29(2), 228. http://dx.doi.org/10.4006/0836-1398-29.2.228

Obande, O. P. (2017a). On the Fundamental Physical Constants: I. Phenomenology. Appl. Phys. Res., 9(5), 42. http://dx.doi.org/10.5539/apr.v9n5p42

Obande, O. P. (2017b). On the Fundamental Physical Constants: II. Field Coupling Geometry. Appl. Phys Res., 9(5), 62. http://dx.doi.org/10.5539.apr.v9n5p62 
Oldershaw, R L. (2007). Discrete Scale Relativity. Astroph. Space Sci., 311(4), 431. http://dx.doi.org/10.1007/ s10509-007-9557-x

Oldershaw, R L. (2014). A Surprising Similarity Between Stars and Atoms. Retrieved from http://www3.amherst. edu/ rloldershaw/OBS.HTML

Pauli, W. (1940). The Connection Betweeb Spin and Statistics. Phys. Rev., 58(8), 716 https://dx.doi.org/10.1103/ PhysRev.58.716

Peebles, P. J. E., \& Ratra, B. (2003). The cosmological constant and dark energy. Rev. Mod. Phys., 75, 559.

Peplow, M. (2013). Planck telescope peers into the primordial universe. Nature News. http://dx.doi.org/10.1038/ Nature.2013.12658

Raghuprasad, P. K. (2013). Planetary spin-orbit attributes in the solar system and their wider implications. Phys. Essays, 26(2), 331.

Redd, N. T. (2017). How big is the Universe? Retrieved from http://www.space.com/24073-how-big-is-theunivese.html

Reiss, A. G. (1998). Evidence that the universe has only recently entered on an era of acceleration out of a previous era of deceleration. Retrieved from http://arxiv.org/abs/astro-ph0104455

Riess, A. G., Macri, L. M., Hoffmann, S. L., Scolnic, D., Casertano, S., Filippenko, A. V., ... \& Chornock, R. (2016). A 2.4\% determination of the local value of the hubble constant Based on observations with the NASA/ESA Hubble Space Telescope, obtained at the Space Telescope Science Institute, which is operated by AURA, Inc., under NASA contract NAS 5-26555. The Astrophysical Journal, 826(1), 56. http://arxiv.org/pdf/1604.0124.pdf

Rubin, V. C. (2000). One hundred years of rotating galaxies. Publ. Astron. Soc. Pacific, $112(772), 747$. http://dx.doi.org/10.1086/316573

Siegel, E. (2017). Why Does The Proton Spin? Physics Hold A Surprising Answer. Retrieved from https://www.forbes.com/sites/startswithabang/2017/04/19/why-does-t...

Slipher, V. M. (1913). The radial velocity of the Andromeda Nebula. Lowell Observatory Bulletin, 2, 56-57. Retrieved from http://adsabs.havard.edu/abs/19131LewOB...2...56S

Sparknotes. (2015). The Most Beautiful Outer Space Image of the Last decade. Retrieved from http://www.sparknotes.com/mindhut/2015/01/12/the-most-beautiful--out...

Spin. (2017). In Wikipedia, the free encyclopedia. Retrieved from https://en.wikipedia.org/wiki/Spin(physcs)20/09/17

Uhlenbeck, G. E., \& Goudsmit, S. (1926). Spinning Electrons and the Structure of Spectra. Nature, 117, 264. Retrieved from https://lorentz.leidenuniv.nl/goudsmit.html

Uhlenbeck, G. E., \& Goudsmit, S. (1926). Spinning Electrons and the Structure of Spectra. Nature, 117, 264. Retrieved from http://lorentz.leidenuniv.nl/goudsmit.html

Wikiversity. (2017). Similarity of matter levels. Retrieved from https://en.wikiversity/wiki/Similarity_of_ matter_levels

\section{Copyrights}

Copyright for this article is retained by the author(s), with first publication rights granted to the journal.

This is an open-access article distributed under the terms and conditions of the Creative Commons Attribution license (http://creativecommons.org/licenses/by/4.0/). 